\title{
Obtención de materiales antifricción hierro-arrabio-latón utilizando residuos
} de virutas de $\operatorname{arrabio}^{(\cdot)}$

\author{
S.N. Nasamov*, N. Krivij ${ }^{* *}$, H.W. Gudenau*, A.I. Babich*, L.L. García** A. Formoso** \\ y A. Cores***
}

Resumen

Las piezas base hierro y acero, obtenidas por pulvimetalurgia, tienen gran utilización en la industria del automóvil y de equipos domésticos. El presente trabajo está dedicado al estudio de las propiedades antifricción de materiales de composición hierro-arrabio-latón, aglomerados mediante prensado y sinterización, obtenidos con mezclas de polvos de hierro, residuos industriales de virutas de arrabio, latón, talco y azufre. Los experimentos fueron hechos utilizando la tecnología de prensado en frío, utilizando una matriz fluida, sin empleo de lubricante sólido. Posteriormente, se realizó una sinterización a temperatura de $1.200^{\circ} \mathrm{C}$ en condiciones isotérmicas, en atmósfera de nitrógeno, en la zona de sinterización durante una hora. Las propiedades físico-mecánicas y antifricción aumentan, en casi dos veces, como resultado del drenaje activo de los gases desde el molde. El estudio de la microestructura de los materiales sinterizados demuestra que existe cementita libre entre los límites de partículas y alrededor de los poros. Se observan grandes aglomeraciones de inclusiones oscuras, compuestas de grafito, zinc y óxidos de hierro, que son centros de tensiones en el material, disminuyendo su resistencia y, por consiguiente, la resistencia a la fricción durante el rozamiento sin lubricante.

Palabras clave Polvos de hierro. Pulvimetalurgia. Sinterización. Materiales compuestos. Residuos.

\section{Antifrictional materials iron-pig iron-brass manufacture using shaving waste products of pig-iron}

Abstract

Keywords

\begin{abstract}
Parts based on iron and steel powders are widely used in the manufacture of automobile and domestic equipment. This work was done to study the anti-friction properties of iron-pig iron-brass compositions of materials which were obtained by pressing and sintering from a mix of iron powders and industrial by products of cast-iron turnings, brass, talc and technical sulphur. Experiments were performed using cold pressure technology in the flowing matrix of the powder composite without solid lubricants. The subsequent sintering was carried out at $1200^{\circ} \mathrm{C}$ under isothermal conditions in a nitrogen atmosphere in the sintering zone during $1 \mathrm{~h}$. The physical-mechanical and anti-friction properties were almost doubled by the active drainage of the gases from the compression mould. The study of the microstructure of the sintered materials showed that free cementite existed between the particle limits and around the pores. Large agglomerations of dark inclusions could be observed, consisting of graphite, zinc and iron oxides, which were points of tension in the material that reduce its durability and, therefore, its wear resistance to dry friction.
\end{abstract}

Iron powders. Powder metallurgy. Sintering. Composites. Waste products.

\section{INTRODUCCIÓN}

Los mercados principales para las piezas fabricadas por pulvimetalurgia son las industrias de automoción y del automóvil ${ }^{[1-3]}$. La densidad y microestructura del material fabricado deben ser optimiza- das especialmente para la fatiga. El desgaste por rozamiento se encuentra en todos los dispositivos con carga cuasiestática, tales como palanca oscilante, guía de válvula, asiento de la válvula y árbol de levas. Según esto, se ha estudiado el comportamiento de los aceros sinterizados muy densos en el

(•) Trabajo recibido el día 21 de mayo de 2002 y aceptado en su forma final el día 27 de enero de 2003.

(*) RWTH Aachen, Institute für Eisenhüttenkunde, Intzestrasse 1, 52056 Aachen, Alemania.

$\left({ }^{* *}\right)$ Centro de Investigaciones Metalúrgicas (CIME), Avda. 51, 23611, Ciudad de La Habana, Cuba.

(***) Centro Nacional de Investigaciones Metalúrgicas (CENIM), Avda. Gregorio del Amo, 8, 28040 Madrid, España. 
Obtención de materiales antifricción hierro-arrabio-latón utilizando residuos de virutas de arrabio

S.N. Nasamov, N. Krivij, H.W. Gudenau, A.I. Babich, L.L. García, A. Formoso y A. Cores

contexto del uso de palanca oscilante, asiento de la válvula y levas ${ }^{[4]}$.

Se ha investigado la obtención de materiales sinterizados resistentes al desgaste por abrasión utilizando mezclas de polvos duros y blandos, por ejemplo, la mezcla de polvos de acero de baja aleación con carburos duros, aceros rápidos, aleaciones patrón o polvos cerámicos ${ }^{[5}$ y 6$]$. La ventaja de la adición de polvos blandos es que la compresibilidad del material permanece alta y mediante la dilución de polvos de acero rápido con polvos de acero de baja aleación, el coste se puede redu$\operatorname{cir}^{[7-9]}$. En estos materiales de composición, consistentes en partículas duras en una matriz blanda, las propiedades del desgaste están determinadas por las partículas duras ${ }^{[10}$ y 11$]$.

Las piezas base hierro y acero, obtenidas por pulvimetalurgia tienen gran utilización en las industrias de automóvil y de equipos domésticos ${ }^{[1-3]}$. A diferencia de las factorías siderúrgicas integradas, que son rentables para capacidades industriales anuales superiores a 1 millón de toneladas, la rentabilidad de la producción de polvos y piezas mediante pulvimetalurgia puede garantizarse desde volúmenes de producción de 100 t/a y el nivel óptimo está dado para una capacidad productiva de 1.000-5.000 t/a, en función del tipo de polvos y artículos elaborados. Estas escalas de producción, relativamente pequeñas, permiten aproximar la capacidad instalada, para el tratamiento de los residuos de virutas a polvos, a las fuentes de formación de estos residuos, es decir, realizar el tratamiento directamente en las empresas que procesan los metales y eliminar completamente las pérdidas por transporte y almacenaje, así como los costos de producción y las pérdidas por oxidación. Los consumos energéticos en la obtención del polvo mediante molienda mecánica de los residuos de virutas, incluyendo las operaciones asociadas, es de $1.500 \mathrm{kWh} / \mathrm{t}$, y en la elaboración de productos laminados comerciales de $4.000-6.000 \mathrm{kWh} / \mathrm{t}^{[12-14]}$.

En la actualidad, cada dos años, la humanidad extrae y consume una mayor cantidad de metal que en los 20 siglos anteriores ${ }^{[15]}$. El tratamiento mecánico de los metales por corte, uno de los más racionales y difundidos métodos de tratamiento, conlleva que en la industria mecánica se acumulen grandes cantidades de virutas. Por ejemplo, en países de la Comunidad de Estados Independientes (CEI) se acumulan anualmente más de 8,5 millones de toneladas de virutas ${ }^{[14]}$.

El procedimiento tradicional para el reciclaje de los residuos de virutas es la fusión, pero durante ésta se produce una pérdida de aproximadamente el $30 \%$ del metal. Sin embargo, el tratamiento de residuos de la industria metalmecánica mediante la pulvimetalurgia, con la finalidad de obtener materiales pulverulentos y piezas a partir de éstos, es una tarea actual de la industria metalúrgica.

Es racional transformar la viruta de arrabio en un polvo metalúrgico mediante molienda ${ }^{[16-18]}$. En la actualidad la producción mundial anual es superior al millar de toneladas ${ }^{[19]}$. Sin embargo, el empleo de la viruta de arrabio después de la molienda no ofrece resultados satisfactorios, debido a los altos contenidos de carbono, silicio y otras impurezas, que ejercen una influencia negativa en los procesos de prensado, sinterización y en las propiedades de los materiales sinterizados. Por consiguiente, previamente es necesario disminuir el contenido total de carbono en el arrabio y eliminar el grafito.

En la industria actual muchas maquinarias presentan altos requerimientos en la seguridad de las piezas, ya que trabajan de forma continua durante largos períodos de tiempo. Estas piezas trabajan en condiciones complejas de fricción y desgaste, bajo la acción de altas presiones y medios agresivos a altas velocidades de operación. En estas condiciones es necesario garantizar un comportamiento eficaz durante los largos períodos de explotación de las maquinas que utilizan estos materiales ${ }^{[20]}$.

Es conocido que la adición de polvo de arrabio en la carga de polvos metalúrgicos para obtener materiales sinterizados, en base hierro, permite obtener materiales aleados con alta resistencia al rozamiento y satisfactorias propiedades físico-mecánicas $^{[17,18}$ y 21$]$.

Muchas piezas de primer orden y componentes de diferentes maquinarias y mecanismos, trabajan en condiciones de rozamiento intensivo. Los materiales resistentes al desgaste empleados en la industria son muy diversos según su composición química. Por ejemplo, hierro-grafito, hierroarrabio, hierro-arrabio-cromo, hierro-vidrio, aleaciones duras, etc. Se emplean ampliamente materiales hierro-grafito y hierro-arrabio. Por procedimientos de pulvimetalurgia se elaboran ruedas dentadas de las más diversas configuraciones y dimensiones sin necesidad de un maquinado complementario. Éstas se elaboran de hierro-grafito, hierro-latón, mezclas de polvos de hierro y de arrabio, y polvos aleados ${ }^{[13]}$. La hipótesis principal, basada en el conocimiento de estos materiales, es la formación de una estructura irregular, que se corresponde con el principio Charpy para materiales 


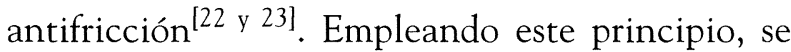
elaboró un material de composición del sistema "hierro-arrabio-cromo", que contiene $25 \%$ de polvo de arrabio y $3 \%$ de cromo. El cromo se introduce en forma de acero aleado $\mathrm{Cr} 30$.

En las producciones por pulvimetalurgia la disminución del costo de obtención de las piezas obtenidas se realiza, en primer lugar, debido la reducción del maquinado posterior. En las piezas simples el costo de tratamiento, en comparación con los materiales fundidos, se reduce como mínimo en un $15 \%$, y en las piezas complejas hasta en un 50 $\%{ }^{[24}$ y 25$]$. Se debe señalar, que la pulvimetalurgia ofrece la posibilidad de obtener materiales de composición del sistema metal-no metal con grandes diferencias según la forma y las dimensiones de las partículas pulverulentas ${ }^{[26-28]}$. Por consiguiente, la elaboración de materiales sobre la base de residuos industriales presenta un especial interés.

En el presente trabajo se fabrican materiales de composición hierro-arrabio-latón por pulvimetalurgia y se estudian sus propiedades antifricción.

\section{PARTE EXPERIMENTAL}

Para fabricar el material hierro-arrabio-latón se han empleado los siguientes materiales:

- polvo de hierro reducido obtenido por atomización (fluidez $30 \mathrm{~s}$; densidad aparente $2,5 \mathrm{~g} / \mathrm{cm}^{3}$; granulometría $\leq 200 \mu \mathrm{m}$ ),

- polvo de latón obtenido por atomización, de composición química, \% en masa: 60,5-63,5, $\mathrm{Cu} ; 0,15$, Fe; 0,08, Pb; el resto, $\mathrm{Zn}$,

- polvo de arrabio especial con grafito puntual interdentrítico, que se obtuvo de residuos de virutas industriales mediante molienda,

- polvo de talco,

- polvo de azufre.

Las virutas de arrabio especial con grafito puntual interdentrítico, contienen los siguientes elementos, \% en masa: 2,0-3,0 C; 2,5-3,8 Si; 0,4-0,9 Mn; 0,1-0,3 P; 0,05-0,1 S. La homogenización de los materiales se realizó en un mezclador, en forma de $\mathbf{Y}$ griega o estrella, durante una hora.

Para la obtención de probetas compactadas, en forma de prisma, de dimensiones $10 \times 10 \times 55$ $\mathrm{mm}$, la carga fue compactada en una prensa modelo HPM-100S, bajo una presión de 400-1.000 MPa, mediante moldes con matriz fluida ${ }^{[27]}$. Con el objetivo de obtener cojinetes de deslizamiento porosos con las propiedades físico-mecánicas necesa- rias, la compactación en frío se realizó en una prensa mecánica "Yosuzuka" con presión nominal de $50 \mathrm{kN}$ según el principio de drenaje activo del aire desde el molde; para garantizar la densidad necesaria para los cojinetes de deslizamiento se utilizan pequeñas presiones de prensado del orden de 100-250 MPa. La velocidad de prensado se estableció manualmente en la escala de 0,1 a $0,45 \mathrm{~m} / \mathrm{s}$; además se establecieron los parámetros siguientes: profundidad de la matriz $22 \mathrm{~mm}$, masa de la carga $16,6 \mathrm{~g}$, y altura de control del prensado $14,6 \mathrm{~mm}$.

Las probetas compactadas, se sinterizaron en un horno de solera corrediza, del tipo "KoyoLindberg", con una velocidad de desplazamiento de la cinta de $3,5 \mathrm{~m} / \mathrm{s}$ y una temperatura de $800-1.200$ ${ }^{\circ} \mathrm{C}$, con retención isotérmica en la zona de sinterización durante $1 \mathrm{~h}$ en atmósfera de nitrógeno. La temperatura en la zona de calentamiento preliminar del horno $\left(575^{\circ} \mathrm{C}\right)$ se controló de forma constante mediante un termopar de cromel-alumel y, en' la zona de sinterización, en dos puntos empleando termopares de platino-platino-rodio. En la zona de enfriamiento del horno, la temperatura se controlaba automáticamente en tres puntos.

A continuación se realiza el calibrado de las probetas sinterizadas, para garantizar la exactitud de las dimensiones y magnitudes permisibles en la tolerancia de los moldes y la interdistribución superficial de las piezas. El calibrado de los cojinetes se realiza después de la sinterización en la misma prensa, en la cual se compactaron. El instrumento de calibración es un molde flexible destinado a calibrar probetas sinterizadas. A diferencia del prensado en molde, en el instrumento de calibración se utiliza una matriz de aleación dura de W-Co. En esta ocasión, se eliminó la posibilidad de formación de desgarramientos en la matriz y en los punzones durante el calibrado de los cojinetes en caso de formarse en la estructura de cementita libre en los limites del grano de metal. La velocidad de calibrado fue constante, $0,25 \mathrm{~m} / \mathrm{s}$, por cuanto no ejerce influencia sobre sus propiedades.

Las características tribológicas de las probetas se estudiaron en una máquina para ensayos de fricción, modelo CMTs-2, empleando un dispositivo de autoinstalación (Fig. 1). Los ensayos tribológicos de los materiales pulverulentos a escala de laboratorio, constituyen uno de los principales índices para valorar su aptitud para trabajar en los pares de fricción. Para realizar los ensayos de estos materiales se utilizan diferentes esquemas de rozamiento, que según la geometría del contacto de la superficie de trabajo pueden ser divididos en dos 


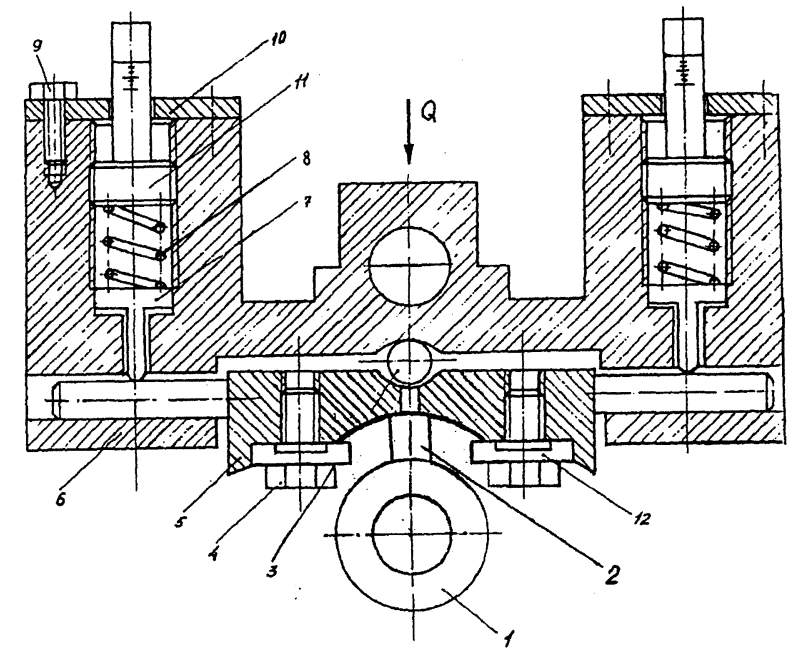

Figura 1. Dispositivo de autoinstalación para la máquina CMTs-2. 1. Contracuerpo; 2. Probeta ensayada; 3. Bola; 4, 9. Tornillo; 5. Base; 6. Cuerpo; 7. Perno desplazable; 8. Muelle; 10. Tapa; 11. Elemento de ajuste; 12. Placa.

Figure 1. Device of self-installation for the machine CMTs-2. 1. Countercoups; 2. Tested probe; 3. Ball; 4, 9. Screw; 5. Base; 6. Body; 7. Moveable bolt; 8. Spring; 10. Lid, 11. Adjustment element; 12. Plate.

grupos: con superficie de contacto nominal constante y variable. El esquema más difundido es el par de fricción cuña-disco, el cual se realiza en la máquina estándar CMTs-2. En muchos casos, es difícil la realización del esquema disco-cuña en máquinas estándar a altas presiones, ya que se requiere que la presión en la zona de contacto no sea superior al limite de fluidez del material de la probeta $^{[29]}$. Para solucionar este problema y realizar una valoración rápida de las propiedades antifricción de los materiales, nosotros hemos desarrollado un nuevo dispositivo adaptable a la máquina CMTs-2 que amplia las posibilidades técnicas del equipo $^{[30]}$. Este dispositivo, por una parte garantiza una autoinstalación libre y uniforme de las probetas bajo carga y, por otra, permite, para fuerzas de rozamiento suficientemente grandes, lograr una aplicación uniforme y compacta de la fuerza en la superficie de rozamiento de las probetas sobre la del contracuerpo. Para excluir la deformación se recomienda un sistema previo de reducción del espesor con un dispositivo cuña-disco de peso $Q$.

La densidad y porosidad de las probetas sinterizadas se determinó mediante un procedimiento hidrostático. La capacidad de absorción de aceite de las probetas impregnadas se determinó mediante pesada de la muestra sinterizada, impregnada en aceite, después de su desengrase. Para realizar los ensayos se utilizó el extractor de Soxhlet y pesas analíticas. Para ello, se pesaron las probetas impregnadas en aceite y a continuación se colocaron en un equipo Soxhlet en el que se realizó la extracción del aceite empleando como diluyente gasolina. Después de eliminar el aceite, la probeta se colocó en una cámara especial a una temperatura de $100-120^{\circ} \mathrm{C}$ durante $1 \mathrm{~h}$, para eliminar los residuos de diluyente que quedaban en los poros de la probeta. Después del secado, la muestra se pesa nuevamente. Repitiendo la extracción y secado, se seguía eliminando aceite hasta que la diferencia en el pesado fuera menor de $0,1 \%$. Posterior al desengrase y secado, la probeta se introduce en parafina fundida y se extrae, se elimina la parafina adherida en la superficie y se pesa suspendida en agua. El contenido de aceite se expresa en función del porcentaje volumétrico de grasa, que fue absorbido por la probeta, mediante la ecuación:

$$
M=\left\{\left(m_{1}-m_{0}\right) /\left(m_{2}-m_{3}\right) d\right\} 100 \%
$$

donde,

M - contenido de aceite, en \% volumétrico

$\mathrm{m}_{1}$ - masa de la probeta impregnada en aceite, $\mathrm{g}$

$\mathrm{m}_{0}$ - masa de la probeta desengrasada, $\mathrm{g}$

$\mathrm{m}_{2}$ - masa de la probeta impregnada con parafina, $\mathrm{g}$

$\mathrm{m}_{3}$ - masa de la probeta impregnada con parafina sumergida en agua, $g$

d - densidad del aceite a temperatura ambiente, $\mathrm{g} / \mathrm{cm}^{3}$

El análisis metalográfico se realizó en el microscopio "Neophot-21". Para determinar la microestructura, los cojinetes se cortaron en forma de probetas especiales en una fresadora. La microestructura de la superficie de los cojinetes se estudio en toda la probeta. El ataque químico de las probetas se realizó en una disolución de $\mathrm{HNO}_{3}$ al $4 \%$.

\section{RESULTADOS}

Los principios para la preparación de materiales pulverulentos resistentes al desgaste son los siguientes ${ }^{[31-33]}$ :

- Bajo la influencia del medio ambiente, los materiales no deben presentar variaciones estructurales, que empeoren las características de resistencia y ductilidad.

- Se recomienda introducir en la composición del material sustancias que permitan trabajar en calidad de lubricante. 
- El coeficiente de fricción entre las inclusiones sólidas y el material de la matriz debe ser mínimo.

Los requerimientos señalados se alcanzaron de forma fácil durante la obtención de materiales mediante la pulvimetalurgia.

La dependencia entre el desgaste relativo y la densidad, se expresa mediante la fórmula empírica siguiente $^{[34]}$ :

$$
X=(9,044-y) / 0,01375
$$

donde,

$\mathrm{X}$ - desgaste relativo, \%

y - densidad del material, $\mathrm{g} / \mathrm{cm}^{3}$

De acuerdo a esta fórmula, el aumento de la densidad en $0,792 \mathrm{~g} / \mathrm{cm}^{3}$ (de 6,986 a $7,778 \mathrm{~g} / \mathrm{cm}^{3}$ ) da la posibilidad de aumentar la resistencia al desgaste de los materiales en 1,7 veces.

Los ensayos realizados muestran que las mejores propiedades tribológicas se observan en los materiales que contienen la composición reflejada en la tabla I.

El coeficiente de fricción en condiciones de rozamiento con lubricante disminuye, tanto con el aumento de la temperatura de sinterización, como con la carga (Fig. 2). Se observa una notable disminución al aumentar la carga de 2 a $4 \mathrm{MPa}$, lo que se explica por el desgaste abrasivo progresivo debido a la baja resistencia del material en estas condiciones. Aproximadamente, por este mismo efecto varía el desgaste de las probetas. El desgaste más bajo se observa en las probetas sinterizadas a $1.200^{\circ} \mathrm{C}$ y ensayadas bajo carga de $2 \mathrm{MPa}$. El desgaste más alto se produce en las probetas que sinterizaron a $1.100{ }^{\circ} \mathrm{C}$ y fueron ensayadas bajo carga de $6 \mathrm{MPa}$ (Fig. 3).

Las propiedades mecánicas y antifricción del material de composición, se encuentran en dependencia directa con el grado de interacción de los componentes durante la sinterización (Tabla II).

Tabla I. Composición del material

Table I. Material composition

\begin{tabular}{ccccc}
\hline \multicolumn{5}{c}{ Polvo de \% en masa: } \\
hierro & arrabio & latón & talco & azufre \\
\hline 33,5 & 54,5 & 10,0 & 1,5 & 0,5 \\
\hline
\end{tabular}

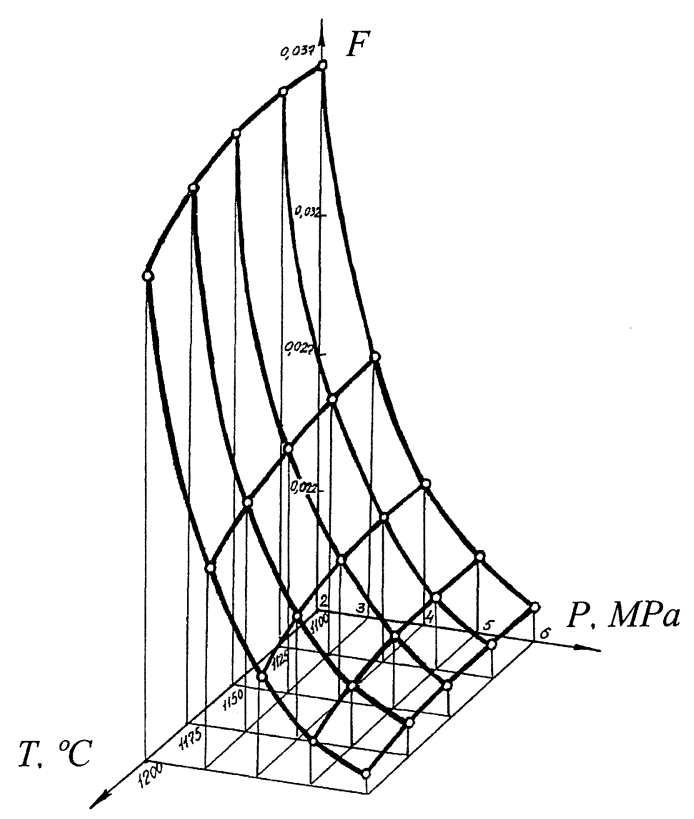

Figura 2. Dependencia entre el coeficiente de fricción $F$, la carga $\mathrm{P}$ y la temperatura de sinterización $\mathrm{T}$ : tiempo de sinterización - $2 \mathrm{~h}$, ensayos de rozamiento con lubricante.

Figure 2. Dependency between the friction coefficient $F$, the load $P$ and the sintering temperature $T$ : sintering time $2 h$, friction with lubricant.

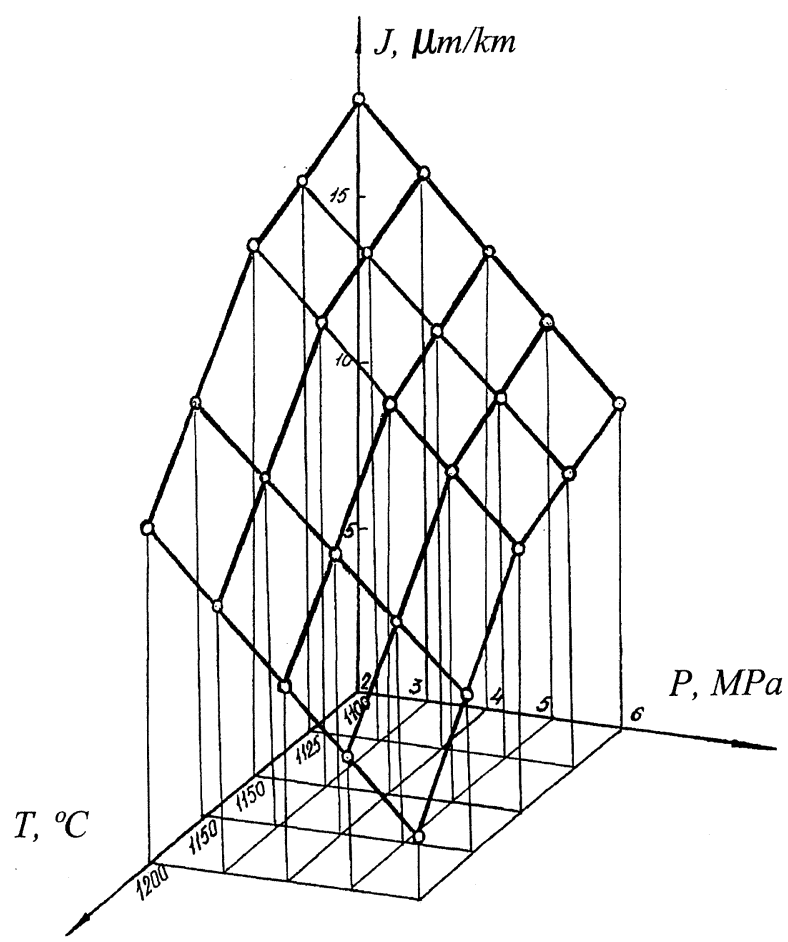

Figura 3. Dependencia entre la intensidad de desgaste (J) del material hierro-arrabio-latón contra la carga (P) y la temperatura de sinterización (T): tiempo de sinterización $2 h$, ensayos de rozamiento con lubricante.

Figure 3. Dependency between wear intensity (J) of iron-cast iron-brass materials against load (P) and the sintering temperature (T): time of sintering - $2 h$, friction with lubricant.

Rev. Metal. Madrid 39 (2003) 164-171 
Obtención de materiales antifricción hierro-arrabio-latón utilizando residuos de virutas de arrabio S.N. Nasamov, N. KriviJ, H.W. Gudenau, A.I. Babich, L.L. García, A. Formoso y A. Cores

Tabla II. Propiedades de los materiales de composición hierro-arrabio-latón obtenidos con prensado en matriz fluida Table II. Properties of iron-pig iron-brass materials, which were obtained with pressed in flowing matrix

\begin{tabular}{|c|c|c|c|c|c|c|c|c|}
\hline \multicolumn{9}{|c|}{ Propiedades del material de composición } \\
\hline $\begin{array}{l}\text { Presión } \\
\mathrm{MPa}\end{array}$ & $\begin{array}{l}\text { Temperatura de } \\
\text { sinterización, }{ }^{\circ} \mathrm{C}\end{array}$ & $\begin{array}{c}\text { Porosidad P } \\
\%\end{array}$ & $\begin{array}{l}\text { Resistencia a la } \\
\text { tracción } \sigma_{\mathrm{B}}, \mathrm{MPa}\end{array}$ & $\begin{array}{l}\text { Resistencia a la } \\
\text { flexión } \sigma_{\mathrm{T}}, \mathrm{MPa}\end{array}$ & $\begin{array}{l}\text { Resilencia KC, } \\
\qquad \mathrm{kJ} / \mathrm{m}^{2}\end{array}$ & $\begin{array}{c}\text { Dureza HB, } \\
\text { MPa }\end{array}$ & $\begin{array}{l}\text { Coeficiente de } \\
\text { fricción, } f\end{array}$ & $\begin{array}{c}\text { Desgaste J, } \\
\mu \mathrm{m} / \mathrm{km}\end{array}$ \\
\hline \multirow{3}{*}{400} & 1.100 & 15,5 & 150 & 242 & 190 & 1.250 & 0,15 & 31 \\
\hline & 1.150 & 14,2 & 158 & 245 & 196 & 1.340 & 0,17 & 28 \\
\hline & 1.200 & 13,2 & 163 & 253 & 201 & 1.390 & 0,17 & 26 \\
\hline \multirow{3}{*}{700} & 1.100 & 9,2 & 201 & 301 & 242 & 1.450 & 0,13 & 14 \\
\hline & 1.150 & 9,0 & 224 & 309 & 256 & 1.520 & 0,12 & 12 \\
\hline & 1.200 & 8,5 & 260 & 312 & 258 & 1.570 & 0,12 & 11 \\
\hline
\end{tabular}

En el caso de prensado de la carga con lubricante, la formación de los contactos mecánicos entre sólidos duros y las partículas "hierro-hierro", "hierro-latón", es menos intensa. Cualquier tipo de contactos directos entre partículas de arrabio, al estar recubiertas con grafito, se dificulta. Por tanto, durante la sinterización se establece un enlace muy débil tanto entre las partículas homogéneas como las heterogéneas y, como resultado, se forma un material con una estructura metálica debilitada. En el caso del prensado en una matriz fluida, sin utilización de lubricante, el drenaje activo de los gases desde el molde, la destrucción de las inclusiones de óxidos en la superficie de las partículas deformadas y la redistribución del grafito en el volumen de prensado, bajo la acción de los esfuerzos del prensado, propician la formación de contactos de alta calidad homogéneos entre las partículas "hierro-hierro" y "hierro-latón". La sinterización de estos compactos se acompaña de una homogenización parcial, contracción y endurecimiento del material de composición. Por ello, estos materiales se caracterizan por sus altas propiedades físico-mecánicas y de antifricción.

El estudio de la microestructura de la aleación hierro-arrabio-latón sinterizada muestra que en todos los casos, en la estructura de los materiales, existe cementita libre entre los limites de las partículas y alrededor de los poros (Fig. 4). Se observan grandes acumulaciones oscuras de inclusiones que están constituidas, fundamentalmente, de grafito, zinc y óxidos de hierro. Estas inclusiones desempeñan la función de concentradores de tensiones en el material, disminuyendo su resistencia y, como consecuencia, la resistencia al desgaste durante el rozamiento sin lubricante.

En la figura 5 se incluye el grafico de dependencia entre la intensidad de desgaste del material hierro-arrabio-latón y la carga durante el rozamiento sin lubricante. La intensidad del desgaste está determinada por el cociente entre el espesor de material desaparecido durante el ensayo de fricción, en $\mu \mathrm{m}$, y el desplazamiento relativo de las superficies que sufren la fricción, en $\mathrm{Km}$. Las probetas fueron prensadas en matriz fluida bajo presión de $1.000 \mathrm{MPa}$ y la sinterización se realizó a 1.200 ${ }^{\circ} \mathrm{C}$. En la figura se observa que con el aumento de la carga, la intensidad de desgaste de las probetas aumenta. Este aumento ocurre también a altas cargas de desgaste $(6-10 \mathrm{MPa})$ y se relaciona con la presencia de latón elástico en el material, que se transporta al contracuerpo, disminuyendo la acción abrasiva de las partículas arrancadas de las zonas de rozamiento.

\section{CONCLUSIONES}

- Se elaboró, a partir de polvos, un material "hierro-arrabio-latón" con la composición siguiente, \%: 33,5 hierro; 54,5 arrabio, 10 latón, 1,5 talco y 0,5 azufre.

- Las mejores propiedades mecánicas y tribológicas de este material se observan para la temperatura de sinterización de $1.200^{\circ} \mathrm{C}$, y se deben al mejoramiento de la interacción de las diversas partículas de arrabio, hierro y la fase líquida del latón.

- Las propiedades físico-mecánicas y de antifricción del material aumentan casi en 2 veces, en el caso de utilizar la tecnología de prensado en frío de la carga en matriz fluida, sin empleo de lubricante y posterior sinterización a temperatura de $1.200^{\circ} \mathrm{C}$ con retención isotérmica en la zona de sinterización durante $1 \mathrm{~h}$ en atmósfera de nitrógeno. La causa fundamental es el drenaje activo de los gases desde el molde. 

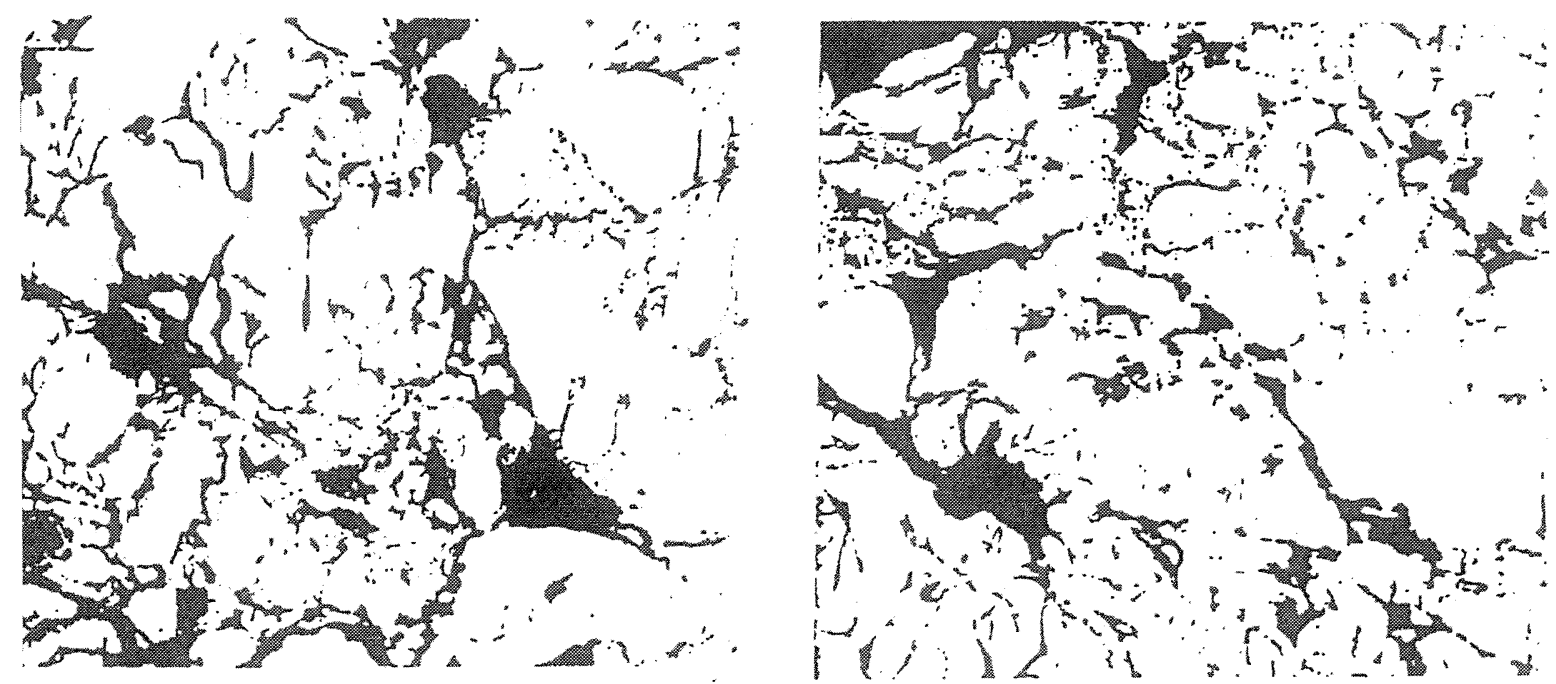

a
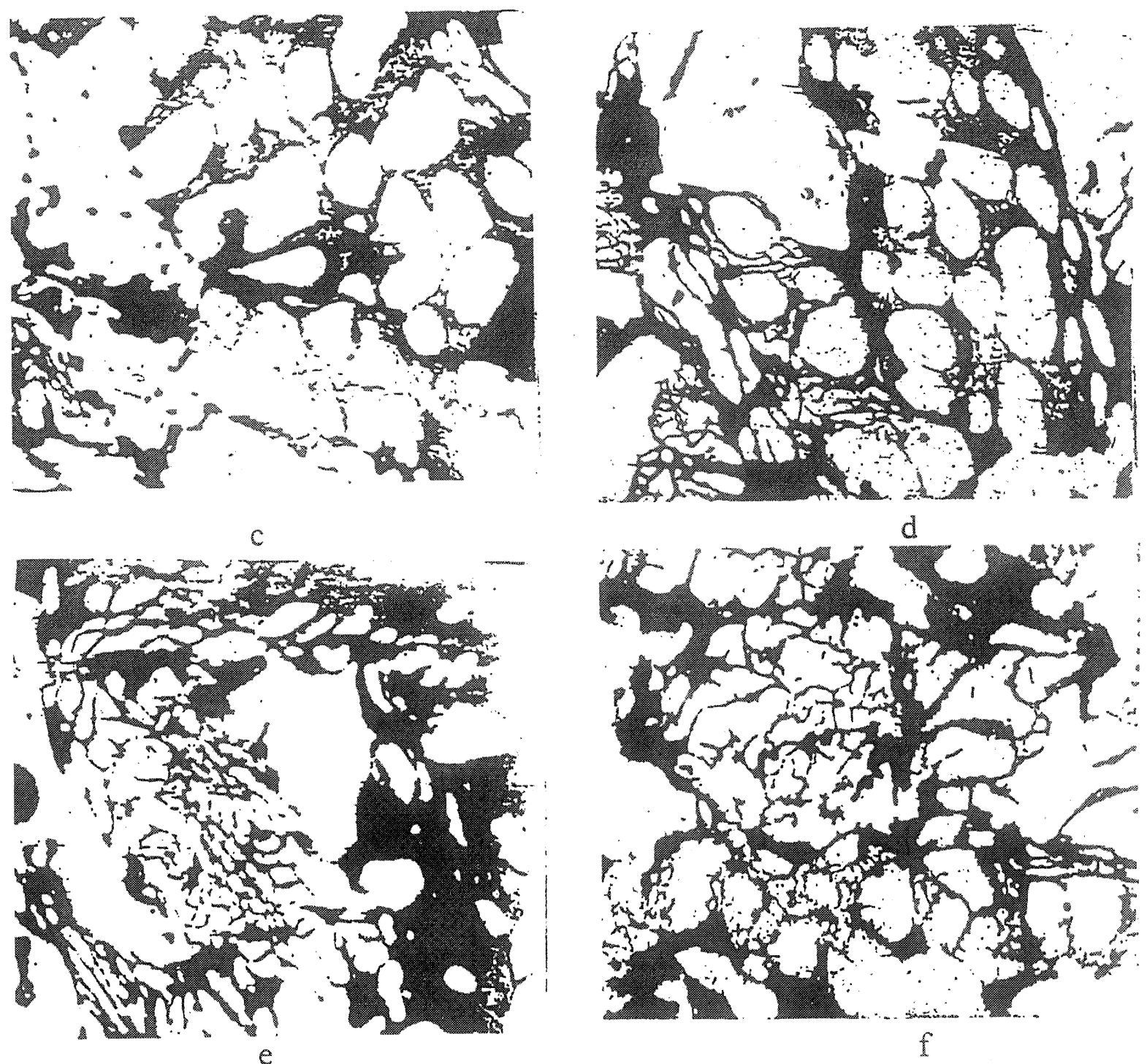

Figura 4. Microestructura de los materiales de hierro-arrabio-latón, $x 200$. Temperatura de sinterización: $a, b=1.110^{\circ} \mathrm{C}$; $c, d=1.150^{\circ} \mathrm{C} ; \mathrm{e}, \mathrm{f}=1.200^{\circ} \mathrm{C}$. Tiempo de sinterización: $\mathrm{a}, \mathrm{c}, \mathrm{e}=1 \mathrm{~h} ; \mathrm{b}, \mathrm{d}, \mathrm{f}=2 \mathrm{~h}$.

Figure 4. Microstructure of iron-cast iron-brass materials, $x 200$. Sintering temperature: $a, b=1110^{\circ} \mathrm{C} ; \mathrm{c}, d=1150{ }^{\circ} \mathrm{C}$; e, $f=1200^{\circ} \mathrm{C}$. Sintering time: $a, c, e=1 h ; b, d, f=2 h$. 
Obtención de materiales antifricción hierro-arrabio-latón utilizando residuos de virutas de arrabio

S.N. Nasamov, N. KriviJ, H.W. Gudenau, A.I. Babich, L.L. García, A. Formoso y A. Cores

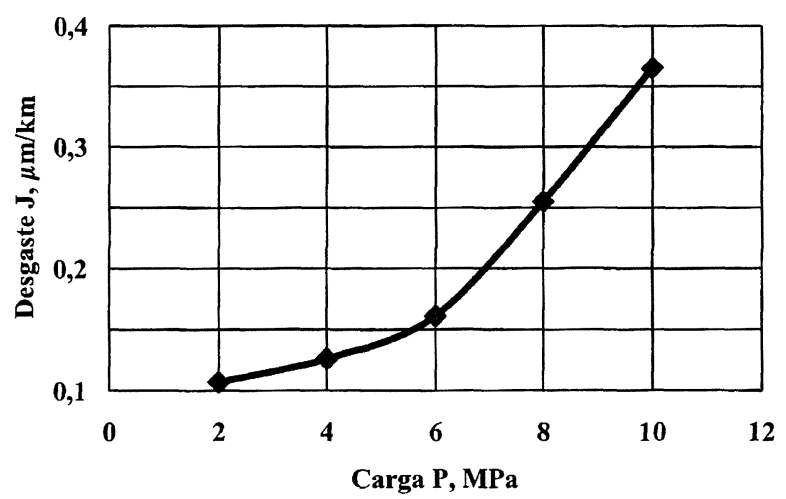

Figura 5. Dependencia entre la intensidad de desgaste del material hierro-arrabio-latón y la carga durante la fricción.

Figure 5. Dependency between the wear intensity of ironcast iron-brass materials and load during the friction.

- El estudio de la microestructura del material sinterizado demuestra que, en todos los casos, en la estructura existe cementita libre en los límites entre las partículas y alrededor de los poros. Se observan grandes acumulaciones oscuras de inclusiones de grafito, zinc y óxidos de hierro. Estas inclusiones desempeñan la función de concentradores de tensiones en el material, disminuyendo su resistencia y, como consecuencia, la resistencia al desgaste durante el rozamiento sin lubricante.

\section{REFERENCIAS}

[1] D. PoHL, Powder Metall. Int. 16 (1984) 70-71.

[2] A. Weaver, Proc. Conf. "Métallurgie des poudres: present et avenir", Grenoble, Francia, Noviembre 1993, SF2M, Paper 3.

[3] Y. MorIoKA, Kawasaki Steel Tech. Report, 29 (1993) 3-13.

[4] B. Martín, C.S. Wright, L. Vicent y A.S. WronsKi, Powder Metall. 42 (1999) 63-69.

[5] M. Hamiudding, Powder Metall. Int. 17 (1985) 20-27.

[6] M. Igharo y J.V. Wood, Powder Metall. 31 (1988) 184-191.

[7] P.R. Brewin, B. Toloui, P.D. Nurthen, J.A. Fellgett, J.V. Wood, M. Igharo, D.S. Coleman y Q. ShaikH, Powder Metall. 32 (1989) 285-290.

[8] M. Igharo y J.V. WoOd, Powder Metall. 33 (1990) 313. 320.

[9] M. Igharo y J.V. WoOd, Powder Metall. 33 (1990) 70-76.

[10] G.L. Zhou y J.V. WoOD, Powder Metall. 38 (1995) 230. 236.
[11] N. Krivij, W. Suwardjo, L. García, A. Cores y A. Formoso, Rev. Metal. Madrid 33 (1997) 229-238.

[12] S.S. KIPARISOV, Poroshk. Metall. 10 (1985) 57-62.

[13] O.V. PODAllKo, La economía en la pulvimetalurgia. Resultados de la ciencia y la técnica, Porozhkovaya Metallurgiya, Moscú, BINITI, T. 2, 1986, pp. 130-184 (en ruso).

[14] V.L. Esikman, S.S. Kiparisov y O.V. Padalko, Poroshk. Metall. 4 (1985) 1-5.

[15] A.F. Belov, Metal: mejora de la calidad. Ciencia y Humanidad, Moscú, Znanie, 1982, pp. 255-269 (en ruso).

[16] S.S. KIPARISOV, Poroshk. Metall. 9 (1979) 56-64.

[17] C. Zhou, J.R. Moon y S. Peacock, Powder Metall. 34 (1991) 33-38.

[18] C. ZHOU y J.R. MoON, Powder Metall. 34 (1991) 205-211.

[19] G.A. GorojovskiI, V.G. ChermnysheV, V.P. Reva y L.V. KovalenKo, Porozhk. Metall. 12 (1988) 1-9.

[20] A.T. Mamedov, Materiales pulverulentos constructivos y antifricción, Baku, Elm, 1991, p. 188 (en ruso).

[21] I.M. Feporchenko, Pulvimetalurgia: Materiales, tecnología, propiedades, campo de aplicación. Manual, Kiev, Naukova Dumka, 1985, p. 624 (en ruso).

[22] B.I. KLIMENKO, Porozhk. Metall. 1 (1962) 80-84.

[23] V.A. Kroja y A.I. BaKavkin, Porozhk. Metall. 1 (1963) 104-109.

[24] P. Jones, K. Buckley-Golder, R. Lawocock y R. SHIVANATH, Int. J. Powder Metall. 33 (1997) 37-44.

[25] W. Schatt y K-P. WIETERS, Pulvermetallurgie: Technologien und Werkstoffe, VDI-Verlag GmHB, Düsseldorf, 1994.

[26] W.J. Huppmann, Wiss. Praxis 9 (1993) 5-35.

[27] C.J. Yu, H.H. Eifert, J. Banhart y J. Baumeister, Mat. Res. Innovat. 2 (1998) 181-188.

[28] P-K. Kar y G.S. Upadhyaya, Powder Metall. Int. 22 (1990) 23-26.

[29] A.T. Mamedov, Tesis Doctoral, Minsk, 1991, p. 503 (en ruso).

[30] S.N. Nasamov. Tesis Doctoral, Bakú, 1991, p. 158 (en ruso).

[31] I.M. Fedorenko, Pulvimetalurgia, Kiev, Naukova Dumka, 1985, p. 624 (en ruso).

[32] M. Martínez, W. Suwardjo, B. Aragón, L. García, A. Formoso y A. Cores, Rev. Metal. Madrid 37 (2001) 597-606.

[33] T.G. GUTOwsKI, Advances Composites Manufacturing, John Wiley (Ed.), 1997, p. 581.

[34] M. Martínez, W. Suwardjo, L. García, A. Formoso y A. Cores, Rev. Metal. Madrid 38 (2002) 173-182. 